\title{
Death-associated Protein Kinase 1 Impairs the Hippocampo- prefrontal Cortical Circuit and Mediates Post-stroke Depression
}

\author{
Xiao $\mathrm{Ke}^{1,2}$, Sehui $\mathrm{Ma}^{1,2}$, Yufen Zhang ${ }^{1,2}$, Yao Yi ${ }^{1,2}$, Hongyan $\mathrm{Yu}^{1,2}$, \\ Dian $\mathrm{Yu}^{1,2}$ and Lei Pei ${ }^{1,2^{*}}$ \\ ${ }^{1}$ Department of Neurobiology, School of Basic Medicine, Tongji Medical College, Huazhong University of Science and Technology, \\ Wuhan 430030, China; ${ }^{2}$ The Institute for Brain Research (IBR), Collaborative Innovation Center for Brain Science, \\ Huazhong University of Science and Technology, Wuhan 430030, China
}

\begin{abstract}
Post-stroke depression (PSD) is a common and complex post-stroke neuropsychiatric disorder, which not only delays functional recovery but also increases mortality, and which currently lacks effective drug therapy. The pathogenesis of PSD is associated with impairment of the subcortical neural circuits and alterations of synaptic plasticity and neurotransmitters, but the exact mechanisms of PSD remain unknown. Our previous work indicates that the death-associated protein kinase 1 (DAPK1) mediates neuronal death after stroke. Genetic deletion of DAPK1 gene or blocking DAPK1 signal in the PSD mouse model can not only alleviate cerebral ischemic injury but also relieve PSD-like behaviors. Our previous work has also demonstrated the following results. First, the neural circuit of dorsal CA1 (dCA1) to medial prefrontal cortex (mPFC) (dCA1-mPFC) is selectively impaired after stroke. Second, the DAPK1 signal is involved in the impairment of dCA1-mPFC neural circuit after stroke. Third, genetic deletion of the DAPK1 gene or blocking of the DAPK1 signal alleviates the injury of dCA1-mPFC neural circuit after stroke and improves PSD-like behaviors. In conclusion, we hypothesize that activated DAPK1 signal after stroke induces apoptosis in the hippocampal dCA1 neurons, leading to loss of the dCA1-mPFC glutamatergic projections, synaptic injury, decrease of glutamate release, inhibition of MPFC neurons, and finally onset of PSD. We hope to further replenish the mechanisms of PSD and provide new insights for PSD treatment.
\end{abstract}

Introduction

In the past three decades, stroke has become a major disease that seriously jeopardizes the health and life of China's residents, and it has placed a heavy burden on patients, their families, and society. According to the newly released 2016 Stroke Epidemiology Report, there are 70 million stroke patients in China, including 2 million new stroke-suffering people per year; moreover, $67.3 \%$ to $80.5 \%$ of the stroke patients represent cases of ischemic stroke. ${ }^{1}$ A recent meta-analysis of 61 cohort studies showed that $31 \%$ of stroke survivors experienced post-stroke depression (PSD) with-

Keywords: DAPK1; Neural circuit; Synaptic injury; Post-stroke depression Abbreviations: CaM, calmodulin; DAPK1, death-associated protein kinase 1; HPA, hypothalamic pituitary adrenal; mPFC, medial prefrontal cortex; PSD, post-stroke depression.

Received: October 23, 2018; Revised: December 21, 2018; Accepted: December 21, 2018

${ }^{*}$ Correspondence to: Lei Pei, Department of Neurobiology, School of Basic Medicine, Tongji Medical College, Huazhong University of Science and Technology, Wuhan 430030, China. E-mail: peilei@hust.edu.cn

How to cite this article: Ke X, Ma S, Zhang Y, Yi Y, Yu H, Yu D, Pei L. Deathassociated Protein Kinase 1 Impairs the Hippocampo-prefrontal Cortical Circuit and Mediates Post-stroke Depression. Exploratory Research and Hypothesis in Medicine 2018;3(4):74-78. doi: 10.14218/ERHM.2018.00018. in 5 years. $^{2}$ PSD is one of the common complications associated with stroke events, and exhibits a series of depressive symptoms and corresponding physical signs. In addition to the neurological deficits, PSD patients also have emotional disorders, such as self-blame and decreased interest. Severe cases may have suicidal thoughts. Delayed, intervention may lead to suicide.

A cohort study of 51,119 PSD cases found that the PSD patients had a significantly higher risk of mortality than healthy people. PSD seriously affects patients' lives and places heavy burdens on their families and society. ${ }^{3}$ Regarding the pathogenesis of PSD, it is considered to be a direct result of specific injury of neuroanatomical structure after stroke or an indirect consequence of negative psychological reactions in patients, and is believed to be the result of a combination of psychological, social and biological factors, such as the severity of stroke, brain damage, sensorimotor dysfunction, and cognitive impairment. ${ }^{4}$ Although the pathogenesis of PSD may be related to impairment of the monoamine system, dysfunction of hypothalamic pituitary adrenal (HPA) axis, destruction of prefrontal cortical circuit, imbalance of glutamate neurotransmitters and proinflammatory factors, the specific neural circuit and molecular mechanisms have not yet been elucidated. ${ }^{5}$

For clinical treatment of PSD, a meta-analysis of a total of 1,655 patients in 16 studies showed that antidepressants produced only a slight benefit, with statistically insignificant difference in 
efficacy. Even worse, antidepressants are liable to increase side effects on the gastrointestinal and central nervous systems, such as confusion, excessive sedation, and tremors. In addition, psychological intervention for PSD is also ineffective. ${ }^{6} \mathrm{PSD}$ is closely related to the patients' poor prognosis, in that it can delay hospital stay, affect recovery of neurological function, and cause loss of independent living ability and even death. However, specific and effective treatments for PSD are unavailable. Therefore, clarifying the pathogenesis of PSD and developing more effective prevention and treatment strategies are of great clinical significance for promoting functional rehabilitation and improving quality of life in PSD patients.

\section{Ischemic stroke causes depression}

In the $1980 \mathrm{~s}$, Robinson et $a .^{7}$ found that reducing the cerebral blood supply in rats could decrease the concentration of catecholamines and hormones, and lead to acute stress response. Due to the decrease of these neurotransmitters and hormones, the rats became slower on the wheel and the animals' willingness to exercise was also reduced. ${ }^{7}$ In 2012, El Husseini et al..$^{8}$ found that most patients with transient ischemic attack developed depression finally, as did stroke patients with functional impairment. A prospective study in China showed that about $30 \%$ of patients had depression at 2 weeks after onset of stroke, and the prevalence of PSD was $31 \%$ at 1 year.

The incidence of PSD is high, and the risk exists for a long time. Fifteen years after stroke, the prevalence of depression is still as high as $31.2 \% .^{9}$ A recent study funded by the Beijing Municipal Science and Technology Commission was conducted with 520 outpatients and hospitalized stroke patients. The incidence of PSD in this group was $34.2 \%$, with mild cases accounting for $20.2 \%$ and moderate cases accounting for $10.4 \%$. The prevalence of PSD was $39 \%$ at 1 month, $53 \%$ at 3-6 months, and $24 \%$ at 1 year after stroke, respectively. If the severity of depression is not distinguished and is collectively referred to as PSD, the incidence is $20-70 \%$. $^{10}$

The above studies show that ischemic stroke can cause depression.

\section{Hippocampal CA1 Injury in PSD}

In ischemic stroke, hippocampal CA1 neurons are more sensitive to ischemic injury than other regions. By using a rodent model of global cerebral ischemia to simulate transient ischemic attacks, a previous study found selective and persistent death of pyramidal neurons in the hippocampal CA1 region, while hippocampal CA3, dentate gyrus, and most cortical neurons were unaffected. Transient cerebral ischemia caused by cardiac arrest or thoracotomy can also cause selective and longer-lasting cell death in the hippocampal CA1 region. ${ }^{11}$ Recent studies have also found that some proteins are abnormally expressed in the hippocampal CA1 region, such as the hamartoma protein Tsc1. ${ }^{12}$ Mitochondrial dysfunction may be another reason for hippocampal CA1 region resistance to ischemia in stroke. ${ }^{13}$ In addition, a mouse model of forebrain ischemia also demonstrated persistent damage to neurons in the hippocampal CA1 area. ${ }^{14}$ Recently, we successfully established a PSD mouse model using light-induced forebrain ischemia, ${ }^{15}$ and we similarly observed degeneration of hippocampal CA1 neurons. The above studies collectively suggest that ischemic stroke can cause selective neuronal damage in the hip- pocampal CA1 area.

Because the hippocampus has wide projections to other brain regions that regulate mood and stress, it is considered to be a critical regulatory site for depression, ${ }^{16}$ and the "hippocampal theory" has been proposed and has supplemented the "monoamine neurotransmitter hypothesis", which is a widely accepted theory for the pathogenesis of depression. Meanwhile, a large number of magnetic resonance imaging studies have found reduced volume of hippocampus in PSD patients. ${ }^{17}$ Animal studies have also shown the reduction of apical dendrites in the hippocampal CA1 neurons. The decreased number of new neurons may be associated with hippocampal volume reduction and depression. ${ }^{18}$ Protecting hippocampal neurons or promoting neurogenesis could relieve symptoms of depression. ${ }^{19}$ Because the pyramidal neurons in the hippocampal CA1 area are mainly glutamatergic neurons, the decrease of glutamate neurotransmitter caused by the loss of pyramidal neurons in the hippocampal CA1 area may lead to the occurrence of PSD. In addition, the hippocampus has a certain inhibitory effect on the activity of the hypothalamic-pituitary-adrenal gland axis. Therefore, inhibition of the HPA axis is weakened after hippocampal injury, ${ }^{20}$ which leads to dysfunction of the HPA axis and induces depression finally. ${ }^{21}$ Thus, damage to the hippocampal CA1 area may be involved in the development of PSD.

\section{Abnormal hippocampo-prefrontal cortical circuit causes de-} pression

Imaging of the patients' brain with depression and histological examination of post-mortem brain have revealed abnormalities in the prefrontal cortex, cingulate gyrus, hippocampus, striatum, and almonds. ${ }^{22}$ At present, many brain regions have been reported to be involved in the pathophysiology of depression. First, deep brain electrical stimulation at the anterior cingulate cortex and nucleus accumbens exhibits an antidepressant-like effect on individuals with refractory depression. This effect is thought to inhibit the activity of the brain region through blockade of the depolarization of axonal fibers. ${ }^{23}$ Second, dopaminergic neural projections in the midbrain (ventral tegmental area to nucleus accumbens) increased activity-dependent release of brain derived neurotrophic factor, which mediates susceptibility to social stress. This effect may be partially produced by phosphorylation of the transcription factor cAMP response element binding protein. ${ }^{24}$ Third, the decreased concentration of neurotrophic factors (such as brain derived neurotrophic factor) reduces the degree of hippocampal nerve regeneration and neuronal processing and complexity. These effects are partly produced by increasing cortisol concentration and decreasing the activity of the cAMP response element binding protein. ${ }^{25}$ Fourth, peripherally released metabolic hormones, such as ghrelin and leptin, can produce mood-related alterations by acting on the hypothalamus and several regions of the limbic system (such as hippocampus, ventral tegmental area, and nucleus accumbens). ${ }^{26}$

In addition, a large number of autopsy and neuro-imaging studies have found that patients with depression have reduced gray matter volume and decreased nerve fiber density in the prefrontal cortex and hippocampus, while the intermediate prefrontal cortex (mPFC) and hippocampus are critical brain regions thought to be involved in depression, ${ }^{27}$ and $\mathrm{mPFC}$ receives abundant synaptic projections from the hippocampal CA1 region. Therefore, it is speculated that the CA1-mPFC circuit participates in the cognitive function of depression. The study also found that $\mathrm{mPFC}$ mainly receives glutamatergic projection fibers from the CA1 area. In the 
rat model of depression, the synaptic plasticity of the CA1-mPFC circuit was also found to be impaired. ${ }^{14,28}$ Our preliminary results also found the loss of dCA1-mPFC projection fibers in the ischemic stroke mouse model. In summary, impaired hippocampalprefrontal circuit caused by ischemic stroke can cause depression.

Ischemic stroke activates the death-associated protein kinase 1 (DAPK1) death signal in the hippocampal CA1 area and induces PSD

Our previous study found that DAPK1 interacts with the excitatory glutamate receptor (GluN2B subunit) and induces neuronal cell death in a mouse model of ischemic stroke, which demonstrates that DAPK1 is a pivotal molecule in neuronal death after ischemic stroke. ${ }^{29,30}$ DAPK1 is a $\mathrm{Ca}^{2+} /$ calmodulin $(\mathrm{CaM})$-dependent serine/ threonine protein kinase, consisting of a kinase domain, a CaMbinding motif, eight repeats of anchored proteins, and a death domain. The CaM-binding motif contains binding sites and a self-inhibition area. ${ }^{31}$ Binding of DAPK 1 to the CaM-binding site results in DAPK1 conformational changes, reactivation of CaM blocked active sites, and DAPK1 activation. ${ }^{32}$ Previous studies have shown that activated DAPK1 is involved in tumor cell death in lymphoma. DAPK1-induced apoptosis has been reported as mainly modulated by Fas, tumor necrosis factor, ${ }^{33}$ apoptotic protease, ${ }^{34}$ and $\mathrm{p} 53 .{ }^{35}$

DAPK1 is associated with nerve injury and aging-related neurological diseases, such as stroke, epilepsy, and Alzheimer's disease. Recently, we reported that DAPK1 activation is involved in the impairments of synaptic transmission, spatial learning and memory. ${ }^{36}$ Notably, DAPK1 also mediates depression-like symptoms in chronic stress. Inhibiting DAPK1 signal by genetic deletion of the DAPK1 gene or pharmacological interruption with an interfering peptide that specifically blocks DAPK1 binding to the GluN2B subunit, both reverse molecular changes and synaptic protein loss caused by chronic stress in the prefrontal cortex, and finally exert rapid and long-lasting antidepressant effects. ${ }^{37}$ In addition, DAPK1 mediates synaptic long-term depression by preventing the binding of $\mathrm{Ca}^{2+} / \mathrm{CaM}$-dependent protein kinase II with GluN2B, which in turn affects learning, and cognitive and emotional functions. ${ }^{38}$ These studies have collectively suggested that the DAPK1 death signal may mediate the development of depression-like symptoms after ischemic stroke.

Recently, we successfully established a PSD mouse model by using light-induced forebrain ischemia, ${ }^{15}$ and further detected protein-protein interactions in the hippocampal CA1 region via mass spectrometry. We found that DAPK1 interacted with caytaxin, which was firstly reported in 2003 by Bomar and his colleagues. ${ }^{39}$ This finding revealed that mutations in the ATCAY gene encoding caytaxin caused Cayman ataxia, an autosomal recessive disorder characterized by poor muscle coordination, mental retardation, loss of head control and eye movements, and difficulties in speaking and walking. In addition, caytaxin is abundantly expressed in neurons of the cortex, cerebellum, hippocampus, olfactory bulb, and basal ganglia. ${ }^{33}$

Akamatsu and colleagues used full-length caytaxin as a bait to perform yeast two-hybridization with mouse adult brain cDNA, ${ }^{40}$ and found that caytaxin can drive the protein light chain of kinesin-1 through its N-terminal ELEWED sequence (amino acids 115-120) and participate in axonal transportation. Kinesin, a key molecule for axonal transportation, can be combined with a variety of linker molecules, such as TRAK1/2 and JIP1, to regulate mitochondrial axonal transportation of amyloid precursor protein. Studies have also shown that caytaxin can act as a linker molecule for kinesin, and transport mitochondria along the axons to neurites, which provide the required energy for various biological processes, such as presynaptic vesicle fusion and neurotransmitter release. ${ }^{34}$ Further, it has been indicated that caytaxin plays an important role in mitochondrial axonal transportation and the synaptic energy supply.

Our recent study demonstrated that DAPK1 interacts with caytaxin, causing apoptosis of hippocampal CA1 neurons. In cultured primary neurons, we employed virus co-transfected DAPK1-related plasmids (activated DAPK1 $(\triangle \mathrm{CaM})$ and inactive DAPK1 (K42A)) with caytaxin. Mitochondria staining showed that $\triangle \mathrm{CaM}$ decreased the number of mitochondria in the neuronal processes, as compared with $\mathrm{K} 42 \mathrm{~A}$. However, co-transfected $\triangle \mathrm{CaM}$ with mutant caytaxin (S46A) showed alleviation of mitochondrial reduction. In addition, co-transfection of $\Delta \mathrm{CaM}$ and wild-type caytaxin caused a relative decrease both in the frequency and amplitude of miniature excitatory post-synaptic currents, suggesting that the serine 46 of caytaxin is essential for DAPK1-induced presynaptic energy deficiency and synaptic dysfunction.

Based on the above studies, we hypothesized that after ischemic stroke, activated DAPK1 causes phosphorylation of the caytaxin protein at serine 46 (pS46), which interrupts the formation of mitochondrial axonal transport complexes, leading to presynaptic mitochondrial reduction, following energy deprivation, then causing impairment of synaptic transmission, and ultimately inducing neuronal apoptosis. Using the conditional DAPK1 knockout $\left(D A P K 1^{-1-}\right)$ mice, we found that the apoptosis of hippocampal dCA1 neurons was decreased and that the depression-like symptoms were effectively alleviated in PSD mice. In addition, the membrane-permeable small molecule polypeptide Tat-CTX composed of caytaxin (43-EDSSSPPST-51) was synthesized to block the binding of the DAPK1 to caytaxin, and to reduce apoptosis of hippocampal dCA1 neurons and alleviate PSD-like symptoms in mice.

\section{Conclusions}

In conclusion, based on previous studies, we hypothesize that activated DAPK1 signal after stroke induces apoptosis in hippocampal dCA1 neurons, which leads to loss of dCA1-mPFC glutamatergic projections, synaptic injury, decrease of glutamate release, inhibition of mPFC neurons, and causes onset of PSD finally. By genetically knocking out the DAPK1 gene $\left(D A P K 1^{-1-}\right)$ or blocking DAPK1 death signal with exogenous peptide may be possible to inhibit the apoptosis of dCA1 neurons and reduce the impairment of dCA1-mPFC neural circuit, thereby alleviating PSD (Fig. 1).

\section{Future research directions}

The proposed hypothesis has important theoretical significance for elucidating the molecular and neural circuit mechanisms of PSD, and will provide new insights for the treatment of PSD. In the future, the following issues need to be further verified. The first is clarifying the molecular mechanism of DAPK1 death signal mediating the decrease of $\mathrm{dCA} 1-\mathrm{mPFC}$ glutamatergic projection after ischemic stroke. The second is revealing the reasons behind the differences between PSD animals and non-PSD animals. And, the last but not least involves information that will benefit the treatment of PSD, such as determining the efficacy and safety of the exogenous peptide Tat-CTX that interferes with the DAPK1 death signal. 


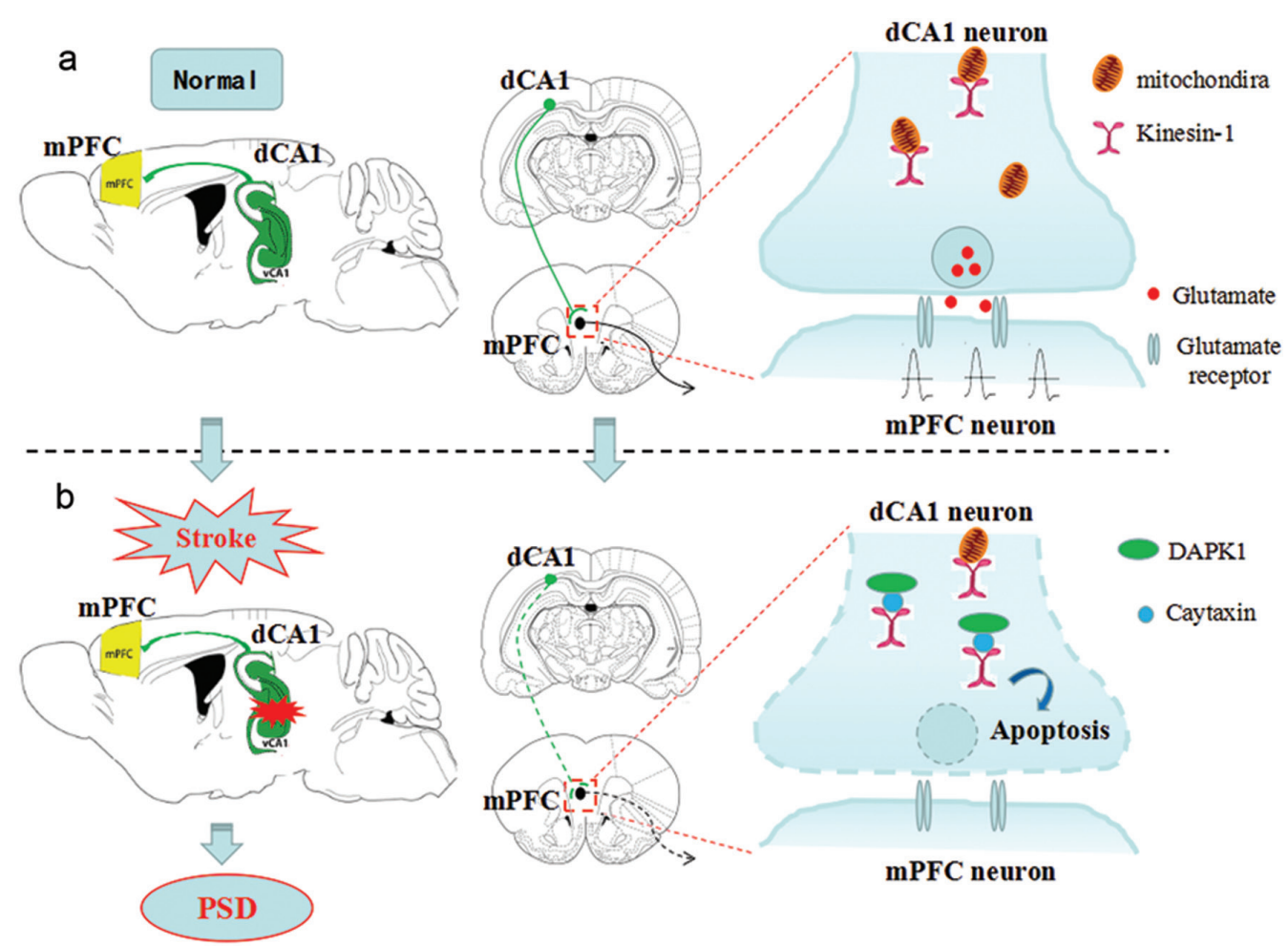

Fig. 1. Schematic diagram of the hypothesis. The neural circuit and molecular mechanism of post-stroke depression (PSD). (a) Normal conditions; (b) Stroke conditions.

\section{Acknowledgments}

This work was supported by the National Natural Science Foundation of China (Grant Nos. 81571078 and 81870932) and the Medjaden Academy \& Research Foundation for Young Scientists (Grant No. MJR20160057).

\section{Conflict of interest}

The authors declare they have no conflict of interests related to this publication.

\section{Author contributions}

Designed the study (LP), drafted the manuscript (XK), contributed to the critical revision of the manuscript for important intellectual content (SM, YZ, YY, HY and DY). All authors approved the version to be published.

\section{References}

[1] Wang W, Jiang B, Sun H, Ru X, Sun D, Wang L, et al. NESS-China investigators: prevalence, incidence, and mortality of stroke in China: results from a nationwide population-based survey of 480687 adults. Circulation 2017;135(8):759-771. doi:10.1161/circulationaha.116.025250.

[2] Bivard A, Lillicrap T, Krishnamurthy V, Holliday E, Attia J, Pagram H, et al. MIDAS (modafinil in debilitating fatigue after stroke): a rand- omized, double-blind, placebo-controlled, cross-over trial. Stroke 2017;48(5):1293-1298. doi:10.1161/strokeaha.116.016293.

[3] Jørgensen TS, Wium-Andersen IK, Wium-Andersen MK, Jørgensen $M B$, Prescott E, Maartensson S, et al. Incidence of depression after stroke, and associated risk factors and mortality outcomes, in a large cohort of danish patients. JAMA Psychiat 2016;73(10):1032-1040. doi:10.1001/jamapsychiatry.2016.1932.

[4] Carson AJ, MacHale S, Allen K, Lawrie SM, Dennis M, House A, et al. Depression after stroke and lesion location: a systematic review. Lancet 2000;356(9224):122-126. doi:10.1016/s0140-6736(00)02448-x.

[5] Robinson RG, Jorge RE. Post-stroke depression: a review. Am J Psychiat 2016;173(3):221-231. doi:10.1176/appi.ajp.2015.15030363.

[6] Kim JS. Management of post-stroke mood and emotional disturbances. Expert Rev Neurother 2017;17(12):1179-1188. doi:10.1080/147 37175.2017.1395281.

[7] Robinson RG, Starr LB, Kubos KL, Price TR. A two-year longitudinal study of post-stroke mood disorders: findings during the initial evaluation. Stroke 1983;14(5):736-741. doi:10.1161/01.str.14.5.736.

[8] El Husseini N, Goldstein LB, Peterson ED, Zhao X, Pan W, Olson DM, et al. Depression and antidepressant use after stroke and transient ischemic attack. Stroke 2012;43(6):1609-1616. doi:10.1161/strokeaha.111.643130.

[9] Sun N, Li QJ, Lv DM, Man J, Liu XS, Sun ML. A survey on 465 patients with post-stroke depression in China. Arch Psychiatr Nurs 2014;28(6):368-371. doi:10.1016/j.apnu.2014.08.007.

[10] Yang $\mathrm{Y}$, Shi $\mathrm{YZ}$, Zhang $\mathrm{N}$, Wang $\mathrm{S}$, Ungvari GS, Ng CH, et al. The disability rate of 5-year post-stroke and its correlation factors: a national survey in China. PLoS One 2016;11(11):e0165341. doi:10.1371/journal.pone. 0165341.

[11] Petito CK, Feldmann E, Pulsinelli WA, Plum F. Delayed hippocampal damage in humans following cardiorespiratory arrest. Neurology 1987;37(8):1281-1286. doi:10.1212/wnl.37.8.1281.

[12] Papadakis M, Hadley G, Xilouri M, Hoyte LC, Nagel S, McMenamin $\mathrm{MM}$, et al. Tsc1 (hamartin) confers neuroprotection against ischemia by inducing autophagy. Nat Med 2013;19(3):351-357. doi:10.1038/ 
$\mathrm{nm} .3097$.

[13] Kurinami H, Shimamura M, Ma T, Qian L, Koizumi K, Park L, et al. Prohibitin viral gene transfer protects hippocampal CA1 neurons from ischemia and ameliorates postischemic hippocampal dysfunction. Stroke 2014;45(4):1131-1138. doi:10.1161/strokeaha.113.003577.

[14] Ouyang YB, Voloboueva LA, Xu LJ, Giffard RG. Selective dysfunction of hippocampal CA1 astrocytes contributes to delayed neuronal damage after transient forebrain ischemia. J Neurosci 2007;27(16):42534260. doi:10.1523/jneurosci.0211-07.2007.

[15] Jin HJ, Pei L, Li YN, Zheng H, Yang S, Wan Y, et al. Alleviative effects of fluoxetine on depressive-like behaviors by epigenetic regulation of BDNF gene transcription in mouse model of post-stroke depression. Sci Rep 2017;7(1):14926. doi:10.1038/s41598-017-13929-5.

[16] McEwen BS. Plasticity of the hippocampus: adaptation to chronic stress and allostatic load. Ann N Y Acad Sci 2001;933:265-277. doi:10.1111/j.1749-6632.2001.tb05830.x.

[17] Lee S, Jeong J, Kwak Y, Park SK. Depression research: where are we now? Mol Brain 2010;3:8. doi:10.1186/1756-6606-3-8.

[18] Videbech P, Ravnkilde B. Hippocampal volume and depression: a meta-analysis of MRI studies. Am J Psychiat 2004;161(11):1957-1966. doi:10.1176/appi.ajp.161.11.1957.

[19] Norrholm SD, Ouimet CC. Altered dendritic spine density in animal models of depression and in response to antidepressant treatment. Synapse 2001;42(3):151-163. doi:10.1002/syn.10006.

[20] Nestler EJ, Barrot M, DiLeone RJ, Eisch AJ, Gold SJ, Monteggia LM. Neurobiology of depression. Neuron 2002;34(1):13-25. doi:10.1016/ s0896-6273(02)00653-0.

[21] Quax RA, Manenschijn L, Koper JW, Hazes JM, Lamberts SW, van Rossum EF, et al. Glucocorticoid sensitivity in health and disease. Nat Rev Endocrinol 2013;9(11):670-686. doi:10.1038/nrendo.2013.183.

[22] Manji HK, Drevets WC, Charney DS. The cellular neurobiology of depression. Nat Med 2001;7(5):541-547. doi:10.1038/87865.

[23] Mayberg HS, Lozano AM, Voon V, McNeely HE, Seminowicz D, Hamani C, et al. Deep brain stimulation for treatment-resistant depression. Neuron 2005;45(5):651-660. doi:10.1016/j.neuron.2005.02.014.

[24] Ressler KJ, Mayberg HS. Targeting abnormal neural circuits in mood and anxiety disorders: from the laboratory to the clinic. Nat Neurosci 2007;10(9):1116-1124. doi:10.1038/nn1944.

[25] Berton O, Nestler EJ. New approaches to antidepressant drug discovery: beyond monoamines. Nature Rev Neurosci 2006;7(2):137-151. doi:10.1038/nrn1846.

[26] Lutter M, Sakata I, Osborne-Lawrence S, Rovinsky SA, Anderson JG, Jung $\mathrm{S}$, et al. The orexigenic hormone ghrelin defends against depressive symptoms of chronic stress. Nat Neurosci 2008;11(7):752-753. doi:10.1038/nn.2139.

[27] Sheline YI. Neuroimaging studies of mood disorder effects on the brain. Biol Psychiatry 2003;54(3):338-352. doi:10.1016/s0006-
3223(03)00347-0.

[28] Zheng C, Zhang T. Synaptic plasticity-related neural oscillations on hippocampus-prefrontal cortex pathway in depression. Neuroscience 2015;292:170-180. doi:10.1016/j.neuroscience.2015.01.071.

[29] Pei L, Shang Y, Jin H, Wang S, Wei N, Yan H, et al. DAPK1-p53 interaction converges necrotic and apoptotic pathways of ischemic neuronal death. J Neurosci 2014;34(19):6546-6556. doi:10.1523/jneurosci.5119-13.2014.

[30] Pei L, Wang S, Jin H, Bi L, Wei N, Yan H, et al. A novel mechanism of spine damages in stroke via DAPK1 and Tau. Cereb Cortex 2015;25(11):4559-4571. doi:10.1093/cercor/bhv096.

[31] Bialik S, Kimchi A. The death-associated protein kinases: structure, function, and beyond. Annu Rev Biochem 2006;75:189-210. doi:10.1146/annurev.biochem.75.103004.142615.

[32] Tu W, Xu X, Peng L, Zhong X, Zhang W, Soundarapandian MM, et al. DAPK1 interaction with NMDA receptor NR2B subunits mediates brain damage in stroke. Cell 2010;140(2):222-234. doi:10.1016/j. cell.2009.12.055.

[33] Cohen O, Inbal B, Kissil JL, Raveh T, Berissi H, Spivak-Kroizaman T, et al. DAP-kinase participates in TNF-alpha- and Fas-induced apoptosis and its function requires the death domain. J Cell Biol 1999;146(1):141148. doi:10.1083/jcb.146.1.141.

[34] Jin Y, Gallagher PJ. Antisense depletion of death-associated protein kinase promotes apoptosis. J Biol Chem 2003;278(51):51587-5193. doi:10.1074/jbc.m309165200.

[35] Raveh T, Droguett G, Horwitz MS, DePinho RA, Kimchi A. DAP kinase activates a p19ARF/p53-mediated apoptotic checkpoint to suppress oncogenic transformation. Nat Cell Biol 2001;3(1):1-7. doi:10.1038/35050500.

[36] Zhu H, Yan H, Tang N, Li X, Pang P, Li H, et al. Impairments of spatial memory in an Alzheimer's disease model via degeneration of hippocampal cholinergic synapses. Nat Commun 2017;8(1):1676. doi:10.1038/s41467-017-01943-0.

[37] Li SX, Han Y, Xu LZ, Yuan K, Zhang RX, Sun CY, et al. Uncoupling DAPK1 from NMDA receptor GluN2B subunit exerts rapid antidepressantlike effects. Mol Psychiatry 2018;23(3):597-608. doi:10.1038/ mp.2017.85.

[38] Goodell DJ, Zaegel V, Coultrap SJ, Hell JW, Bayer KU. DAPK1 mediates LTD by making CaMKII/GluN2B binding LTP specific. Cell Rep 2017;19(11):2231-2243. doi:10.1016/j.celrep.2017.05.068.

[39] Bomar JM, Benke PJ, Slattery EL, Puttagunta R, Taylor LP, Seong E, et al. Mutations in a novel gene encoding a CRAL-TRIO domain cause human Cayman ataxia and ataxia/dystonia in the jittery mouse. Nat Genet 2003;35(3):264-269. doi:10.1038/ng1255.

[40] Akamatsu R, Ishida-Kitagawa N, Aoyama T, Oka C, Kawaichi M. BNIP-2 binds phosphatidylserine, localizes to vesicles, and is transported by Kinesin-1-1. Genes Cells 2015;20(2):135-152. doi:10.1111/gtc.12209. 